\title{
Magnetic and magnetocaloric properties of the $\mathrm{La}_{0.9-x} \mathrm{Ag}_{x} \mathrm{Mn}_{1.1} \mathrm{O}_{3}$ compounds
}

\author{
E. Zubov ${ }^{1,2}$, A. Pashchenko ${ }^{3}$, N. Nedelko $^{4}$, I. Radelytskiy ${ }^{4}$, K. Dyakonov $^{5}$, A. Krzyżewski $^{4}$, \\ A. Ślawska-Waniewska ${ }^{4}$, V. Dyakonov ${ }^{4}$, and H. Szymczak ${ }^{4}$ \\ ${ }^{1}$ G.V. Kurdyumov Institute for Metal Physics, NASU, 36 Acad. Vernadsky Boulevard, Kyiv 03680, Ukraine \\ ${ }^{2}$ Vasyl' Stus Donetsk National University, Vinnytsia 021021, Ukraine \\ E-mail: eezubov@ukr.net \\ ${ }^{3}$ O.O. Galkin Donetsk Institute of Physics and Engineering, 46 Nauki Ave., Kyiv 03680, Ukraine \\ ${ }^{4}$ Institute of Physics, PAS, Al. Lotników 32/46, Warsaw 02-668, Poland \\ ${ }^{5}$ Ioffe Physico-Technical Institute RAS, S.-Petersburg 194021, Russia
}

Received February 28, 2017, published online August 27, 2017

\begin{abstract}
The influence of $\mathrm{Ag}^{+}$ions and $\mathrm{Mn}$ non-stoichiometry on the magnetic properties and magnetocaloric effect in the $\mathrm{La}_{0.9-x} \mathrm{Ag}_{x} \mathrm{Mn}_{1.1} \mathrm{O}_{3}$ compounds has been studied. The $\mathrm{Ag}^{+}$ions content substituting for trivalent lanthanum ions was varied from $x=0.05$ to 0.3. Magnetic measurements have been performed over a wide temperature (350-5 K) and magnetic field (up to $5 \mathrm{~T}$ ) ranges. All the manganites studied show the ferromagnetic-like ordering with a first order magnetic phase transition. The magnetic entropy changes were evaluated from the isothermal curves of magnetization versus an applied magnetic field using both the thermodynamic Maxwell relation (MR) and calculation based on the Landau theory of phase transitions. The largest values of the magnetocaloric effect as well as the relative cooling power among the studied manganites are equal to $5.76 \mathrm{~J} /(\mathrm{kg} \cdot \mathrm{K})$ and $185 \mathrm{~J} / \mathrm{kg}$, respectively, for $\mathrm{La}_{0.7} \mathrm{Ag}_{0.2} \mathrm{Mn}_{1.1} \mathrm{O}_{3}$ in the magnetic field change of $5 \mathrm{~T}$ near the Curie temperature of $T_{C}=271 \mathrm{~K}$. A good correlation between the magnetization, colossal magnetoresistance and magnetic entropy changes as a function of $\mathrm{Ag}$ concentration in the $\mathrm{La}_{0.9-x} \mathrm{Ag}_{x} \mathrm{Mn}_{1.1} \mathrm{O}_{3}$ manganites was established.
\end{abstract}

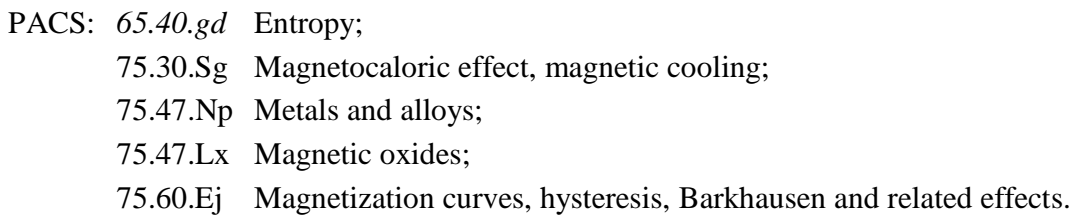

Keywords: $\mathrm{La}_{0.9-} \mathrm{Ag}_{x} \mathrm{Mn}_{1.1} \mathrm{O}_{3}$ manganites, magnetization, magnetic entropy, magnetocaloric effect, magnetoresistance.

\section{Introduction}

In recent years, there has been increasing interest to use manganites not only as materials with colossal magnetoresistance, but also as materials with promising magnetocaloric properties.

Most studies have been carried out on the self-doped $\mathrm{La}_{1-x} \mathrm{Mn}_{1+\chi} \mathrm{O}_{3}$ manganites or $\mathrm{La}_{1-x} \mathrm{~A}_{x} \mathrm{MnO}_{3}$ manganites with stoichiometric content of manganese doped with two valence A cations, where $\mathrm{A}$ are the alkaline-earth ions $\left(\mathrm{Ca}^{2+}, \mathrm{Sr}^{2+}, \ldots\right)[1,2]$. The investigations have shown high enough magnetocaloric effect (MCE) in these compounds. The largest reported value of the magnetocaloric effect in $\mathrm{La}_{1-x} \mathrm{Ca}_{x} \mathrm{MnO}_{3}$ was found to be $6.25 \mathrm{~J} /(\mathrm{kg} \cdot \mathrm{K})$ at $216 \mathrm{~K}$ upon magnetic field change of $1 \mathrm{~T}$ for $x=0.3$ which exhibits a first order paramagnetic-ferromagnetic transition [3].

Similar to divalent A cations, monovalent cations can also change the valence of manganese. Indeed, it has been observed that monovalent cations (like sodium, potassium or silver) are able to induce ferromagnetism in $\mathrm{LaMnO}_{3}$ with the Curie temperatures ranging from 180 to $340 \mathrm{~K}$ [4-7] and to lead to large magnetocaloric effects. At non- 
isovalent replacement of A cation an electric neutrality can be achieved not only by changing valence of manganese $\left(\mathrm{Mn}^{3+} \rightarrow \mathrm{Mn}^{4+}\right)$, but also by structural defects connected with formation of anion vacancies. Such mechanism of charge compensation caused by anion vacancies is of particular interest in the study of manganites at replacement of A cation by monovalent ions.

In a series of papers, an influence of replacement of three valence La cation by monovalent ions, such as $\mathrm{K}^{+}[8,9]$ or $\mathrm{Na}^{+}[10]$, on the magnetic behavior of manganites has been studied. The lanthanide manganites doped with monovalent cations like sodium or potassium display significant magnetic entropy change induced by the applied magnetic field. The studies of $\mathrm{La}_{1-x} \mathrm{~K}_{x} \mathrm{MnO}_{3}(0<x<0.15)$ reveal that the maximum magnetic entropy change $\left(\Delta S_{\max }\right)$ is increased with increasing potassium content from $2.73 \mathrm{~J} /(\mathrm{kg} \cdot \mathrm{K})$ at $260 \mathrm{~K}$ for $x=0.05$ to $3 \mathrm{~J} /(\mathrm{kg} \cdot \mathrm{K})$ at $309 \mathrm{~K}$ for $x=0.15$ under $H=1 \mathrm{~T}$ [8]. The maximum value of MCE at 500 Oe was found to vary from $\Delta T=0.017 \mathrm{~K}$ for $\mathrm{La}_{0.95} \mathrm{~K}_{0.05} \mathrm{MnO}_{3}$ to $\Delta T=0.107 \mathrm{~K}$ for $\mathrm{La}_{0.87} \mathrm{~K}_{0.13} \mathrm{MnO}_{3}$ [9].

The study of magnetic properties of $\mathrm{La}_{1-x} \mathrm{Na}_{x} \mathrm{MnO}_{3}$ samples ( $x=0.08-0.16$ ) has shown that at low temperatures all the samples are ferromagnetic and the temperature of transition from paramagnetic to ferromagnetic state increases with increasing sodium content [10]. For the $\mathrm{La}_{0.8} \mathrm{Na}_{0.2} \mathrm{MnO}_{3}$ sample, the $\left(\Delta S_{\max }\right)$ value is found to be $0.43 \mathrm{~J} /(\mathrm{kg} \cdot \mathrm{K})$ at $333 \mathrm{~K}$ in a magnetic field change of $1 \mathrm{~T}$ [11]. The magnetocaloric properties reported for $\mathrm{La}_{1-x} \mathrm{Na}_{x} \mathrm{MnO}_{3}(x=0-0.15)$ show that the magnetic entropy change reaches the highest value of $2.178 \mathrm{~J} /(\mathrm{kg} \cdot \mathrm{K})$ at $298 \mathrm{~K}$ at the applied magnetic field of $1 \mathrm{~T}$ for $x=0.1$ [12].

The $\mathrm{La}_{1-x} \mathrm{Ag}_{x} \mathrm{MnO}_{3}$ manganites are the example of such group of compounds in the case of the substitution of $\mathrm{La}^{3+}$ ions for monovalence ions [13]. A replacement of $\mathrm{La}$ ions by $\mathrm{Ag}^{+}$ions should lead both to the mixed valence states of manganese ions $\left(\mathrm{Mn}^{3+}\right.$ and $\left.\mathrm{Mn}^{4+}\right)$ and intrinsic inhomogeneities connected with the occurrence of anion and cation vacancies. According to the principle of electric neutrality, at substitution of trivalent lanthanum for monovalent $\mathrm{Ag}^{+}$ions a charge compensation occurs due to increase of the average valence of manganese ions, i.e., a part of manganese ions in the $\mathrm{B}$ positions becomes tetravalent $\left(\mathrm{Mn}^{3+} \rightarrow \mathrm{Mn}^{4+}\right)$. This should surely cause a change of magnetic properties of the $\mathrm{La}_{1-x} \mathrm{Ag}_{x} \mathrm{MnO}_{3}$ manganites as a result of the competition between antiferromagnetic superexchange and ferromagnetic double-exchange interactions.

The measurements of magnetic and magnetocaloric properties of Ag-doped lanthanum manganites were presented in a few reports [14-16]. For example, the magnetocaloric effect in the polycrystalline $\mathrm{La}_{0.8} \mathrm{Ag}_{0.2} \mathrm{MnO}_{3}$ perovskite-type manganite was found to be considerably higher than in other perovskite compounds, in which La is substituted for divalent alkali-earth elements. The large magnetic entropy change produced by the abrupt increase of effective magnetic moment is associated with a first- order phase transition at the Curie temperature [14,15]. For the $\mathrm{La}_{0.85} \mathrm{Ag}_{0.15} \mathrm{MnO}_{3}$ sample, the maximum magnetic entropy change value is found to be $4.2 \mathrm{~J} /(\mathrm{kg} \cdot \mathrm{K})$ at $280 \mathrm{~K}$ in an applied magnetic field of $2.6 \mathrm{~T}$ [16]. The manganites studied have showed almost the same relative cooling power (RCP) values equal to about $100 \mathrm{~J} / \mathrm{kg}$ for a field change of $2.6 \mathrm{~T}$. For the $\mathrm{La}_{0.7} \mathrm{Ag}_{0.3} \mathrm{MnO}_{3}$ sample, the $\Delta S_{\max }$ is found to be $1.35 \mathrm{~J} /(\mathrm{kg} \cdot \mathrm{K})$ in a magnetic field change of $1 \mathrm{~T}$ around the room temperature [14].

In this work, the influence of $\mathrm{Ag}^{+}$ions substituting trivalent lanthanum ions and non-stoichiometric Mn ions on magnetization and magnetocaloric effect is presented. For this, the $\mathrm{La}_{0.9-\gamma} \mathrm{Ag}_{x} \mathrm{Mn}_{1+\delta} \mathrm{O}_{3}$ compounds with a silver content variation from $x=0.05$ to 0.3 and at excess manganese of $\delta=0.1$ have been studied. The manganites studied are exhibited to undergo paramagnetic-ferromagnetic phase transitions. An increase of Ag content slightly shifts the $T_{C}$ to high-temperature region. An excessive Mn content is shown to lower the field cooled magnetization of manganites studied. The magnetocaloric effect was evaluated from the isothermal curves of spontaneous magnetization versus an applied magnetic field using both the thermodynamic Maxwell's relation and calculation based on the Landau theory. The $\left(-\Delta S_{\max }\right)$ values determined by both methods agree. It is found that the excessive Mn negligible influences on the MCE magnitude. The correlation between the magnetization, colossal magnetoresistance and magnetic entropy changes as a function of $\mathrm{Ag}$ ions content in the $\mathrm{La}_{0.9-x} \mathrm{Ag}_{x} \mathrm{Mn}_{1.1} \mathrm{O}_{3}$ manganites was established.

\section{Samples and experimental}

The ceramic samples with general formula $\mathrm{La}_{0.9-x} \mathrm{Ag}_{x} \mathrm{Mn}_{1.1} \mathrm{O}_{3}(x=0.05,0.1,0.15 .0 .2,0.3)$ have been studied. The bulk polycrystalline samples prepared by mixing $\mathrm{La}(\mathrm{OH})_{3}, \mathrm{AgNO}_{3}$ and $\mathrm{Mn}_{3} \mathrm{O}_{4}$ powders in the desired proportions were synthesized using the standard solidphase synthesis technique at a high temperature. In order to keep the oxygen stoichiometry the reactions were carried out in silica tubes. The powder mixture was heat-treated at temperature of $900^{\circ} \mathrm{C}$ (for $20 \mathrm{~h}$ ) with intermediate grinding of as-obtained products. The obtained powders were compacted under pressure into pellets (of about $1 \mathrm{~mm}$ thickness) and sintered at $1050^{\circ} \mathrm{C}$ in air for $20 \mathrm{~h}$. Finally, these pellets were slowly cooled down to the room temperature in the air along with the furnace.

To study the structure and magnetic properties the following experimental methods were used, namely: a) room temperature $\mathrm{x}$-ray diffraction method using the $\mathrm{Cu} K_{\alpha}$ radiation, b) method of dc magnetization in the temperature interval of $5-350 \mathrm{~K}$ and in magnetic field up to $H=5 \mathrm{~T}$ using a PPMS magnetometer, and c) four-probe method to determine the magnetoresistance effect $\mathrm{MR}=\left(\rho-\rho_{H}\right) / \rho$, where $\rho(T)$ and $\rho H(T)$ are the temperature dependences of resistance in zero and $5 \mathrm{~T}$ magnetic fields, respectively, 
over a temperature range of $77-400 \mathrm{~K}$. The oxygen content was determined by the gravimetric and iodometric methods. The field change was slow enough (with field steps of $2 \mathrm{kOe}$ ) to consider all magnetization processes as isothermal. The magnetization was measured with temperature steps of 2-3 K over a temperature range around the magnetic phase transition.

\section{Structural properties}

According to $\mathrm{x}$-ray diffraction data all the samples have a perovskite-like rhombohedral structure (Table 1). The samples with concentration $x<0.15$ are single phase. When $x \geq 0.15$ the increased traces of $\mathrm{Mn}_{3} \mathrm{O}_{4}$ phase were observed. The chemical composition of these powders checked with room temperature $x$-ray diffraction method is close to the molar one of $\mathrm{La}_{0.9-} \mathrm{Ag}_{x} \mathrm{Mn}_{1.1} \mathrm{O}_{3}$.

As is seen in Table 1 , the unit cell $a$ parameter in rhombohedral installation monotonically increases with increasing $x$. It is caused by the replacement of $\mathrm{La}^{3+}$ ions with ionic radius of $R_{\mathrm{La}^{3+}}{ }_{\{12\}}=1.50 \AA$ (coordination number $\mathrm{CN}=12$ ) [17] by $\mathrm{Ag}^{+}$ions with larger ionic radius

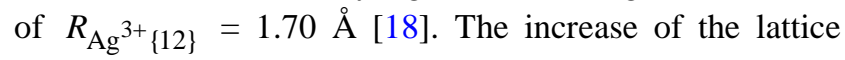
constant allows to propose that the $\mathrm{Mn}-\mathrm{O}$ bond length has a tendency to increase with increasing Ag content.

\section{Magnetic properties}

We now discuss the magnetic properties of the samples studied. The temperature dependences of field-cooled magnetization $\left(M_{F C}\right)$ for manganites with various Ag content (from $x=0.05$ to 0.3 ) measured in a magnetic field of 100 Oe are presented in Fig. 1.

Figure 1 displays the effect of Ag content on the magnetic behavior of the samples studied. As is seen in the inset (b) of Fig. 1, the $M_{F C}$ magnetization changes anomalously as Ag content is increased, namely, $M_{F C}$ increases

Table 1. Type of symmetry, lattice parameters of the perovskite structure and phase composition of the $\mathrm{La}_{0.9-x} \mathrm{Ag}_{x} \mathrm{Mn}_{1.1} \mathrm{O}_{3}$ ceramics in the rhombohedral $(Z=8)$ installation $(Z$ is the number of formula units per unit cell)

\begin{tabular}{|c|c|c|c|c|}
\hline \multirow[t]{2}{*}{$x$} & \multirow[t]{2}{*}{$\begin{array}{c}\text { Type of } \\
\text { symmetry }\end{array}$} & \multicolumn{2}{|c|}{$\begin{array}{c}\text { Rhombohedral } \\
\text { installation, } \\
\text { lines (440) and }(04 \overline{4})\end{array}$} & \multirow[t]{2}{*}{ Second phase } \\
\hline & & $a, \AA$ & $\alpha, \operatorname{deg}$ & \\
\hline 0 & \multirow[t]{6}{*}{$R \overline{3} c$} & 7.813 & 90.48 & Single phase \\
\hline 0.05 & & 7.823 & 90.50 & Single phase \\
\hline 0.1 & & 7s.833 & 90.52 & Single phase \\
\hline 0.15 & & 7.842 & 90.54 & $\mathrm{Mn}_{3} \mathrm{O}_{4}<3 \%$ \\
\hline 0.2 & & 7.848 & 90.53 & $\mathrm{Mn}_{3} \mathrm{O}_{4}<3 \%$ \\
\hline 0.3 & & 7.850 & 90.48 & $\mathrm{Mn}_{3} \mathrm{O}_{4}<3 \%$ \\
\hline
\end{tabular}

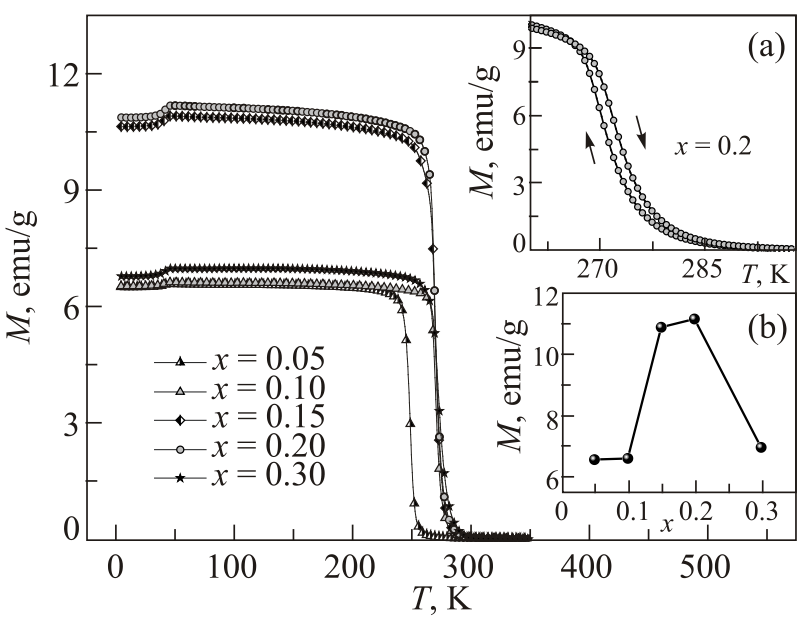

Fig. 1. The temperature dependences of field-cooled magnetization for manganites with various Ag content measured in a magnetic field of 100 Oe. The inset (a) shows a thermal hysteresis of $M(T)$ curves near the $T_{C}$ temperature for the sample with $x=0.2$. The inset (b) shows the $M_{F C}(x)$ dependence at $T=50 \mathrm{~K}$ for $x$ variation from 0.05 to 0.3 .

with changing $x$ from 0.05 to 0.2 and then decreases at $x>0.2$. An increase of $M_{F C}$ is connected with growth of number of holes $\left(\mathrm{Mn}^{4+}\right)$. The ratio of $\mathrm{Mn}^{4+} / \mathrm{Mn}^{3+}$ approaches a maximum value at Ag content of $x=0.2$. The increase of the $\mathrm{Mn}^{4+}$ fraction, suggesting the increase of number of $\mathrm{Mn}^{3+} / \mathrm{Mn}^{4+}$ pairs, leads to the enhancement of the double exchange interaction and causes the $M_{F C}$ magnetization to rise. At $x \geq 0.3$, the samples magnetization has almost the same value because the $\mathrm{Mn}^{4+}$ fraction does not change due to the segregation of Ag into metal clusters.

It should be noted that the low-temperature magnetization of the non-stoichiometric manganites with $x=0.05-0.2$ is lower as compared to the $M_{F C}$ magnetization of manganites with stoichiometric content [13].

Anomaly of magnetization observed on the $M(T)$ curves at temperature of about $42 \mathrm{~K}$ (Fig. 1) means that in homogeneous ferromagnetic phase a small amount of antiferromagnetic phase of $\mathrm{Mn}_{3} \mathrm{O}_{4}$ exists. It agrees with the x-ray diffraction data.

The character of FC magnetization curves as a function of temperature in the applied magnetic field of 100 Oe (Fig. 1) shows that all investigated samples display a paramagnetic-ferromagnetic phase transition. The $M_{F C}(T)$ curves demonstrate a large steepness and a thermal hysteresis $\left(\Delta T_{C}=1.6-1.9 \mathrm{~K}\right)$ near the Curie temperature, $T_{C}$, that are typical of a first order transitions. As an example, a thermal hysteresis of $M(T)$ curves equal to $\Delta T_{C}=1.8 \mathrm{~K}$ near $T_{C}=271 \mathrm{~K}$ for the sample with $x=0.2$ is shown in the inset (a) of Fig. 1.

The $d M / d T$ curves allow to determine the ferromagnetic ordering temperatures. An increase of Ag content slightly shifts the $T_{C}$ to high-temperature region. The minimum Curie temperature obtained is equal to $250 \mathrm{~K}$ for the sample with $x=0.05$. As is seen in Fig. 1 and Table 2, the $T_{C}$ 
Table 2. Parameters of the Curie-Weiss law $\chi=\chi_{0}+C /(T-\theta)$ obtained by fitting to experimental susceptibility $H / M$ for different content $x$ of Ag in the temperature range from 210 to $360 \mathrm{~K}$ and the Curie temperature, $T_{C}$. The effective number of Bohr magnetons, $n_{\text {eff }}=g \sqrt{S(S+1)}$, and magnetic moment $\mu$ obtained from saturation magnetization at $5 \mathrm{~K}$

\begin{tabular}{c|c|c|c|c|c|c}
\hline \hline$x$ & $\chi_{0}$ & $\mathrm{C}$ & $\begin{array}{c}\Theta, \\
\mathrm{K}\end{array}$ & $n_{\mathrm{eff}}$ & $\begin{array}{c}\mu, \\
\mu_{B} / \mathrm{Mn}\end{array}$ & $\begin{array}{c}T_{C}, \\
\mathrm{~K}\end{array}$ \\
\hline \hline 0.05 & -0.001 & 5.06 & 250.3 & 6.4 & 3.48 & 250 \\
0.1 & -0.004 & 5.04 & 267.4 & 6.4 & 3.38 & 270 \\
0.15 & -0.004 & 4.65 & 268.3 & 6.1 & 3.21 & 270 \\
0.2 & -0.0016 & 4.27 & 269.4 & 5.9 & 3.08 & 271 \\
0.3 & -0.0001 & 3.63 & 271.7 & 5.4 & 2.78 & 272 \\
\hline \hline
\end{tabular}

temperature increases up to $272 \mathrm{~K}$ with increasing Ag content up to 0.3. However, in Ref. 14 it is shown that the $T_{C}$ temperature determined for $\mathrm{La}_{0.7} \mathrm{Ag}_{0.3} \mathrm{MnO}_{3}$ is $306 \mathrm{~K}$. For the same Ag concentration studied in Ref. 19 the Curie temperature is equal to $293 \mathrm{~K}$. This discrepancy could be due to the fact that the actual Ag contents might be different in the samples studied.

The analysis of high-temperature susceptibilities $(\mathrm{M} / \mathrm{H})$ was performed using the Curie-Weiss (CW) law:

$$
\chi(T)=\frac{C}{T-\theta},
$$

where the Curie constant $C=3 k_{B} / \mu_{\text {eff }}^{2}, \quad \mu_{\text {eff }}(S)=$ $=\mu_{B} g \sqrt{S(S+1)}$ is the effective magnetic moment and $\theta$ the paramagnetic $\mathrm{CW}$ temperature. The fitting of the experimental $(H / M)(T)$ dependences to the linear function $1 / \chi(T)$ at high temperatures has shown that the temperature dependences of the inverse susceptibility are linear for the paramagnetic phase and obey the CW law. The $\theta$ temperatures were calculated as a result of the fitting in the temperature range from 260 to $350 \mathrm{~K}$, when $\mathrm{Ag}$ content increases from $x=0.05$ to $x=0.3$, respectively (Table 2). They have a positive sign that is indicative of dominant ferromagnetic interactions.

From the magnetic field dependences of magnetization $M(H)$ (at $H=5 \mathrm{~T}$ and $T=5 \mathrm{~K}$ ) the saturation FM moment, $\mu$, is shown to decrease from 3.48 to $2.78 \mu_{B} / \mathrm{Mn}$ with increasing Ag concentration from $x=0.05$ to $x=0.3$, respectively (Table 2). The experimental value of the saturation FM moment is lower than the theoretical value of $3.6 \mu_{B} / \mathrm{Mn}$ calculated for complete spin alignment of the Mn magnetic moments in manganites without excess manganese for $x=0$.

\section{Magnetocaloric effect}

The magnetic entropy changes $\left(\Delta S_{M}\right)$ due to changes of magnetic field applied to the system, characterizing the magnetocaloric effects, were determined from the isothermal magnetization curves for temperature interval from 320 to $210 \mathrm{~K}$ and in a magnetic field range up to $5 \mathrm{~T}$.
To analyze MCE in the studied manganites with a first order phase transition close to a second order one, the calculation method actual for a 2nd order transition was employed. The temperature dependence of $\Delta S_{M}$ was calculated using the thermodynamic Maxwell relation (MR) [20]. According to MR, the total magnetic entropy change, $\Delta S_{M}(T, H)$, of the magnetic system during the magnetization processes in a magnetic field change of $H$ is given by relation

$$
\Delta S_{M}(T, H)=S_{M}(T, H)-S_{M}(T, 0)=\int_{0}^{H_{\max }}\left(\frac{\partial M}{\partial T}\right)_{H} d H,
$$

where $T, H_{\max }$ and $M$ are the temperature, maximum external field and magnetization, respectively. A numerical calculation of $\Delta S_{M}(T, H)$ was performed using the expression :

$$
\Delta S_{M}(T, \Delta H)=\sum\left\{\left(M_{i+1}-M_{i}\right) /\left(T_{i+1}-T_{i}\right)\right\} \Delta H_{i},
$$

where $M_{i+1}$ and $M_{i}$ are the experimental values of magnetization at $T_{i+1}$ and $T_{i}$ temperatures, respectively, under an applied magnetic field $H_{i}$ [21].

A comparison of the calculated maximum magnetic entropy changes, $-\Delta S_{M \max }$, corresponding to magnetic fields of $1,3,4$ and $5 \mathrm{~T}$ for the $\mathrm{La}_{0.9-x} \mathrm{Ag}_{x} \mathrm{Mn}_{1.1} \mathrm{O}_{3}$ samples with $x=0.05,0.1,0.15,0.2$ and 0.3 is presented in Table 3 .

The largest values of magnetocaloric effect among the studied manganites induced by the magnetic fields of $5 \mathrm{~T}$ and $1 \mathrm{~T}$ are equal to 5.76 and $2.46 \mathrm{~J} /(\mathrm{kg} \cdot \mathrm{K})$ for $\mathrm{La}_{0.7} \mathrm{Ag}_{0.2} \mathrm{Mn}_{1.1} \mathrm{O}_{3}$, respectively. These results are close to the $-\Delta S_{M \text { max }}$ values of $4.92 \mathrm{~J} /(\mathrm{kg} \cdot \mathrm{K})$ [22] and $3.4 \mathrm{~J} /(\mathrm{kg} \cdot \mathrm{K})$ [14] under magnetic field changes of 5 and $1 \mathrm{~T}$, respectively.

As an example, the $-\Delta S_{M}(T, H)$ dependences for the $\mathrm{La}_{0.7} \mathrm{Ag}_{0.2} \mathrm{Mn}_{1.1} \mathrm{O}_{3}$ sample around the PM-FM transition in a magnetic field changes of $H=1$ and $5 \mathrm{~T}$ are presented in Fig. 2.

As it is seen in Table 3 , the $\left(-\Delta S_{M \max }\right)$ value increases with changing $x$ from 0.05 to $x=0.2$ and then is reduced at $x>0.2$ that correlates well with the behavior of magnetization as a function of $x$ shown in the inset (b) of Fig. 1. This observation is indicative of similar influence of partial sub-

Table 3. Maximum magnetic entropy changes, $-\Delta S_{M}$, as a function of $\mathrm{Ag}$ concentration for the $\mathrm{La}_{0.9-x} \mathrm{Ag}_{x} \mathrm{Mn}_{1.1} \mathrm{O}_{3}$ compounds under magnetic field changes of $H=1,3,4$ and $5 \mathrm{~T}$

\begin{tabular}{c|c|c|c|c}
\hline \hline$x$ & $\begin{array}{c}-\Delta S_{\max }(1 \mathrm{~T}), \\
\mathrm{J} /(\mathrm{kg} \cdot \mathrm{K})\end{array}$ & $\begin{array}{c}-\Delta S_{\max }(3 \mathrm{~T}), \\
\mathrm{J} /(\mathrm{kg} \cdot \mathrm{K})\end{array}$ & $\begin{array}{c}-\Delta S_{\max }(4 \mathrm{~T}), \\
\mathrm{J} /(\mathrm{kg} \cdot \mathrm{K})\end{array}$ & $\begin{array}{c}-\Delta S_{\max }(5 \mathrm{~T}), \\
\mathrm{J} /(\mathrm{kg} \cdot \mathrm{K})\end{array}$ \\
\hline \hline 0.05 & 1.81 & 3.78 & 4.43 & 4.98 \\
0.1 & 2.01 & 4.03 & 4.66 & 5.21 \\
0.15 & 2.30 & 4.36 & 5.00 & 5.54 \\
0.2 & 2.46 & 4.57 & 5.20 & 5.76 \\
0.3 & 1.92 & 3.80 & 4.38 & 4.88 \\
\hline \hline
\end{tabular}




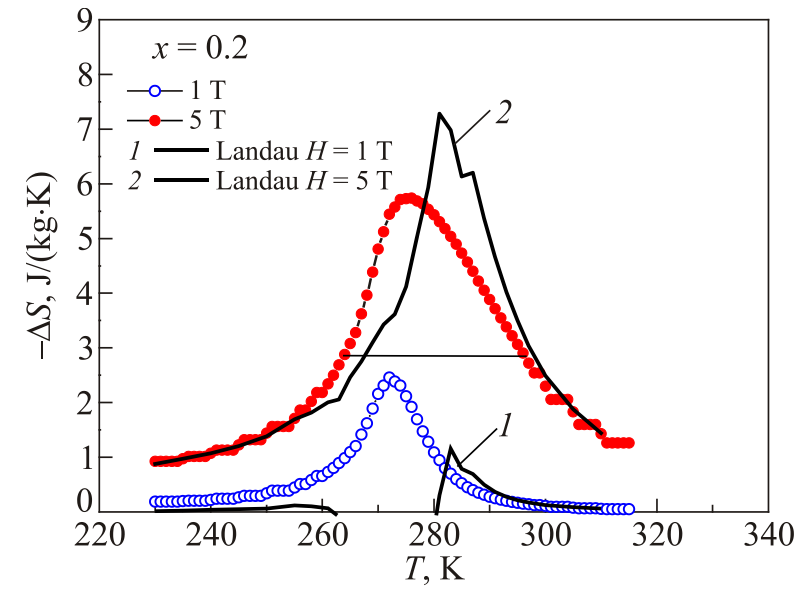

Fig. 2. Magnetic entropy change $\left(-\Delta S_{M}\right)$ as a function of temperature for the $\mathrm{La}_{0.7} \mathrm{Ag}_{0.2} \mathrm{Mn}_{1.1} \mathrm{O}_{3}$ sample under magnetic field changes of $\Delta H=1$ and $5 \mathrm{~T}$. Temperature dependences of $-\Delta S_{M}$ for the $\mathrm{La}_{0.7} \mathrm{Ag}_{0.2} \mathrm{Mn}_{1.1} \mathrm{O}_{3}$ sample in 1 and $5 \mathrm{~T}$ magnetic fields (solid lines 1 and 2, respectively) calculated using the Landau theory.

stitution of Ag for La on magnetic and magnetocaloric properties. This influence can be explained by the fact that $\mathrm{Ag}^{+}$ions introduced into the perovskite phase substitute $\mathrm{La}^{3+}$ ions causing some of $\mathrm{Mn}^{3+}$ ions to become $\mathrm{Mn}^{4+}$ ions proportional to the substituted Ag ion concentration. An increase of Ag content in perovskite phase increases the $\mathrm{Mn}^{4+} / \mathrm{Mn}^{3+}$ ratio since one $\mathrm{Ag}^{+}$ion oxidizes two $\mathrm{Mn}^{3+}$ ions to two $\mathrm{Mn}^{4+}$ ions, which is in favor of magnetic entropy change [13]. It should be noted that the excessive Mn ions have negligible influence on the MCE magnitude. It agrees with data obtained in Ref. 23.

The evolution of magnetic entropy change as a function of temperature was also analyzed based on the Landau theory of phase transitions [23,24]. The magnetic free energy, $F(T, M)$, as functions of magnetization and temperature can be expressed as follows [25]:

$$
F(T, M)=\frac{1}{2} \alpha(T) M^{2}+\frac{1}{4} \beta(T) M^{4}-M H,
$$

where $\alpha(T)$ and $\beta(T)$ are the temperature dependent Landau coefficients, which provide information about magnetoelastic coupling and electron-electron interaction [26].

From the condition of equilibrium, we obtain the equation

$$
\frac{H}{M}=\alpha(T)+\beta(T) M^{2} .
$$

The $\alpha(T)$ and $\beta(T)$ coefficients are determined by linear fitting of $H / M$ function from Eq. (5) to corresponding experimental Arrott plots. In accordance with the Landau theory the variation of isothermal entropy for a field change from 0 to $H$ has the form

$$
\begin{gathered}
-\Delta S_{T}(T, \Delta H)=S_{T}(T, 0)-S_{T}(T, H)= \\
=\frac{1}{2} \alpha^{\prime}(T)\left(M^{2}-M_{0}^{2}\right)+\frac{1}{4} \beta^{\prime}(T)\left(M^{4}-M_{0}^{4}\right),
\end{gathered}
$$

where $\alpha^{\prime}(T)$ and $\beta^{\prime}(T)$ are the temperature derivatives of the Landau coefficients. At $T<T_{C}$ we have $M_{0}^{2}=-\alpha(T) / \beta(T)$ and for $T>T_{C}$ the spontaneous magnetization $M_{0}^{2}=0$. The $\alpha^{\prime}(T)$ and $\beta^{\prime}(T)$ derivatives are determined by linear interpolation of the $\alpha(T)$ and $\beta(T)$ dependences.

The temperature dependences of $-\Delta S_{M}$ for the $\mathrm{La}_{0.7} \mathrm{Ag}_{0.2} \mathrm{Mn}_{1.1} \mathrm{O}_{3}$ sample in a magnetic field changes of 1 and 5 T presented in Fig. 2 by solid lines 1 and 2, respectively, have been calculated using Eq. (6) based on the Landau theory of phase transitions. It is seen that the MCE values evaluated by both ways are in agreement.

It is interesting to consider the relative cooling power (RCP) which can be determined from the following expression [27]:

$$
\operatorname{RCP}(S)=\Delta S_{\max } \Delta T_{F W H M},
$$

where $\Delta S_{\max }$ is the peak value of $\Delta S_{M}(T)$ and $\Delta T_{F W H M}$ is the full width at half the peak of $\Delta S_{M}(T)$. The RCP(S) value for $x=0.2$ in an applied magnetic field of $5 \mathrm{~T}$ is equal to $185 \mathrm{~J} / \mathrm{kg}$. It should be noted that because of a small quantity of thermal hysteresis near the Curie temperature at calculation of the relative cooling power a magnetic losses connected with hysteresis were neglected. The known RCP values for the $\mathrm{La}_{1-x} \mathrm{Ag}_{x} \mathrm{MnO}_{3}$ samples are of the same order of magnitude as calculated RCP value. It should be noted that the RCP values exhibit a linear dependence on an applied magnetic field that agrees with data obtained in Ref. 22.

The studied manganites are of interest since they belong

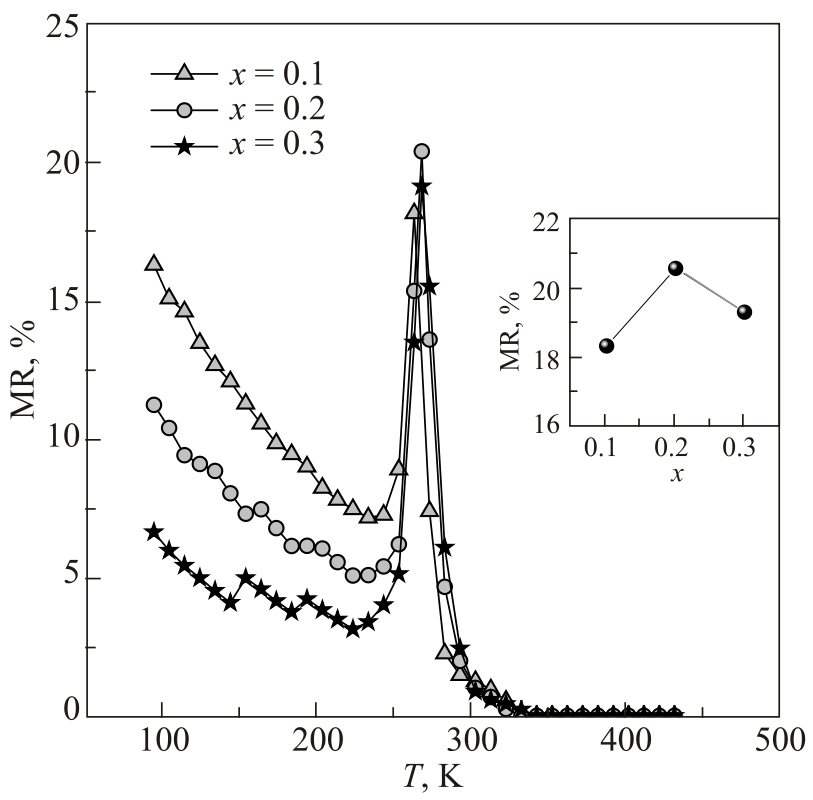

Fig. 3. Concentration dependences of magnetoresistance effect for the $\mathrm{La}_{0.9-x} \mathrm{Ag}_{x} \mathrm{Mn}_{1.1} \mathrm{O}_{3}$ manganites. 
to materials with high magnetoresistance effect (MRE). The magnetoresistance of a series of bulk polycrystalline $\mathrm{La}_{0.9-x} \mathrm{Ag}_{x} \mathrm{Mn}_{1.1} \mathrm{O}_{3}$ samples was investigated (Fig. 3). The temperature dependence of MRE shows that the MRE peak is observed near the metal-semiconductor and ferromagnet-paramagnet phase transitions. As is seen in Fig. 3, the magnetoresistance value increases with changing $x$ from 0.1 to 0.2 and then is decreased at $x>0.2$. Thus, there is a good agreement between the largest value of MRE and maximum magnetic entropy changes. It is known [28] that an electron-phonon interaction influences strongly on magnetic properties of manganites. In particular, the manganites with a strong electron-phonon interaction show high enough MRE. As rule, the alkaline-earth ions doped perovskites have a strong electron-phonon interaction and intensive MCE. Apparently, the optimal relation between the contents of free charge carriers and electron-phonon binding is achieved at the Ag ion concentration of $x=0.2$.

The results of complex studies performed on the $\mathrm{La}_{0.9-x} \mathrm{Ag}_{x} \mathrm{Mn}_{1.1} \mathrm{O}_{3}$ manganites show a certain correlation between the magnetization (Fig. 1), magnetoresistance effect (Fig. 3) and magnetic entropy changes (Fig. 2) as a function of Ag content.

\section{Conclusion}

In this paper, the results of measurements of magnetic and magnetocaloric properties of the $\mathrm{La}_{0.9-x} \mathrm{Ag}_{x} \mathrm{Mn}_{1.1} \mathrm{O}_{3}$ manganites containing the excessive $\mathrm{Mn}$ content are presented. The $\mathrm{Ag}^{+}$ions content substituting for trivalent lanthanum ions was varied from $x=0.05$ to 0.3 . Magnetic measurements have been performed over a wide temperature (350-5 K) and magnetic field (up to $5 \mathrm{~T}$ ) ranges. The studies have shown that partly replacement of La ions by Ag ones leads to occurrence of the ferromagnetic state. The manganites studied demonstrate a large steepness and a thermal hysteresis of $M(T)$ curves in the vicinity of magnetic phase transitions, which are characteristic for a first order transitions. The magnetic entropy changes were evaluated from the isothermal curves of magnetization versus an applied magnetic field using both the thermodynamic Maxwell relation (MR) and calculation based on the Landau theory of phase transitions. The MCE values evaluated by both methods are in agreement. The largest values of the magnetocaloric effect as well as the relative cooling power among the studied manganites calculated using MR are equal to $5.76 \mathrm{~J} /(\mathrm{kg} \cdot \mathrm{K})$ and $185 \mathrm{~J} / \mathrm{kg}$, respectively, for $\mathrm{La}_{0.7} \mathrm{Ag}_{0.2} \mathrm{Mn}_{1.1} \mathrm{O}_{3}$ in a magnetic field change of $5 \mathrm{~T}$ near the Curie temperature of $T_{C}=271 \mathrm{~K}$. A good correlation between the magnetization, magnetoresistance and magnetic entropy changes as a function of Ag concentration in the $\mathrm{La}_{0.9-X} \mathrm{Ag}_{x} \mathrm{Mn}_{1.1} \mathrm{O}_{3}$ manganites was established. The large relative cooling power for $\mathrm{La}_{0.7} \mathrm{Ag}_{0.2} \mathrm{Mn}_{1.1} \mathrm{O}_{3}$ makes these materials one of the most promising candidates as active refrigerants for magnetic refrigeration technology near room temperature.

\section{Acknowledgments}

This work was performed in the laboratories cofinanced by the ERDF Project POIG.02.02.00-00-025/09. This study was partially supported by the National Center for Research and Development, research project No. PBS2/A5/36/2013.

The study was carried out within the Fundamental Research Programme funded by the MES of Ukraine (Project No. 0117U002360).

1. J.M.D. Coey, M. Viret, and von S. Molnar, Adv. Phys. 48, 167 (1999).

2. V. Markovich, A. Wisniewski, and H. Szymczak, Handbook Magn. Mater. 22, 1 (2014).

3. A.N. Ulyanov, J.S. Kim, G.M. Shin, Y.M. Kang, and S.I. Yoo, J. Phys. D 40, 123 (2007).

4. T. Diehl, P. Chandouet, J.C. Joubert, and J. Pierre, J. Appl. Phys. 81, 4970 (1997).

5. M.M. Savosta, V.A. Borodin, P. Novak, Z. Jirak, J. Hejtmanek, and M. Marysko, Phys. Rev. B 57, 13379 (1998).

6. W. Zhong, W. Chen, W.P. Ding, N. Zhang, A. Hu, Y.W. Du, and Q.J. Yan, Eur. Phys. J. B 3, 169 (1998).

7. T. Tao, Q.Q. Cao, K.M. Gu, H.Y. Xu, S.Y. Zhang, and Y.W. Du, Appl. Phys. Lett. 77, 723 (2000).

8. S. Das and T.K. Dey, J. Alloys Comp. 440, 30 (2007).

9. A.M. Aliev, A.G. Gamzatov, A.B. Batdalov, A.S. Mankevich, and I.E. Korsakov, JETP 112, 460 (2011).

10. A.I. Tovstolytkin, V.M. Tsmots, L.I Pankiv, P.G. Litovchenko, and I.S. Pankiv, Fiz. Nizk. Temp. 36, 220 (2010) [Low Temp. Phys. 36, 280 (2010)].

11. D.-L. Hou, C.-X. Yue, Y. Bai, Q.-H. Liu, X.-Y. Zhao, and G.-D. Tang, Solid State Commun. 140, 459 (2006).

12. S. Das and T.K. Dey, J. Phys. D: Appl. Phys. 40, 1855 (2007).

13. A. EkberIrmak, A. Coskun, E. Tasarkuyu, S. Akturk, G. Unlu, Yu. Samancioglu, C. Sarikurkcu, B.M. Kaynar, and A. Yucel, J. Magn. Magn. Mater. 322, 945 (2010).

14. T. Tang, K.M. Gu, Q.Q. Cao, D.H. Wang, S.Y. Zhang, and Y.W. Du, J. Magn. Magn. Mater. 222, 110 (2000).

15. N.T. Hien and N.P. Thuy, Physica B 319, 168 (2002).

16. K. Kamilov, A.G. Gamzatov, A.M. Aliev, A.B. Batdalov, A.A. Aliverdiev, Sh.B. Abdulvagidov, O.V. Melnikov, O.Yu. Gorbenko, and A.R. Kaul, J. Phys. D: Appl. Phys. 40, 4413 (2007).

17. R.D. Shannon, Acta Cryst. A 32, 751 (1976).

18. L.A. Groat, J.L. Jambor, and B.C. Pemberton, Can. Mineral. 41, 921 (2003).

19. M. Battabyal and T.K. Dey, Physica B 367, 40 (2005).

20. V.K. Pecharsky and K.A. Gschneidner, Jr., J. Magn. Magn. Mater. 200, 44(1999). 
21. R.D. McMichael, J.J. Ritter, and R.D. Shull, J. Appl. Phys. 73, 6946 (1993).

22. M. Koubaa, Y. Regaieg, W. Cheikhrouhou Koubaa, A. Cheikhrouhou, S. Ammar-Merah, and F. Herbst, J. Magn. Magn. Mater. 323, 252 (2011).

23. G.F. Wang, Z.R. Zhao, L.R. Li, and X.F. Zhang, J. Magn. Magn. Mater. 397, 198 (2016).

24. L.D. Landau and E.M. Lifshitz, Statistical Physics, Pergamon Press, New York (1958).
25. J.S. Amaral, M.S. Reis, V.S. Amaral, T.M. Mendonca, J.P. Araujo, M.A. Sa, P.B. Tavares, and J.M. Vieira, J. Magn. Magn. Mater. 290, 686 (2005).

26. W.J. Lu, X. Luo, C.Y. Hao, W.H. Song, and Y.P. Sun, J. Appl. Phys. 104, 113908 (2008).

27. L. Caron, M. Hudl, V. Höglin, N.H. Dung, C.P. Gomez, M. Sahlberg, E. Brück, Y. Andersson, and P. Nordblad, Phys. Rev. B 88, 094440 (2013).

28. D.M. Edwards, Adv. Phys. 51, 1259 (2002). 